\title{
THE KÁRMÁN-TSIEN PRESSURE-VOLUME RELATION IN THE TWO-DIMENSIONAL SUPERSONIC FLOW OF COMPRESSIBLE FLUIDS*
}

\author{
BY \\ N. COBURN \\ University of Texas
}

1. Introduction. T. v. Kármán and H.S. Tsien ${ }^{1}$ have treated the two-dimensional subsonic flow of a perfect, irrotational, compressible fluid by replacing the adiabatic pressure-volume curve by the tangent line drawn at an arbitrary point of this curve.

First, we shall discuss the applicability of the Kármán-Tsien idea in the supersonic range. Secondly, we shall show that when the Kármán-Tsien relation can be used (fairly uniform completely supersonic flow), the characteristics form a Tschebyscheff net (fish net). ${ }^{2}$ However, we shall be concerned with those regions of the physical plane which can be mapped into a Tschebyscheff net in a unique one-to-one manner. Hence, we shall not study the onset of shock. Further, we shall show that if the diagonal curves of the net of characteristics are drawn so as to correspond to equidistant values of the arc length parameter along the characteristics, then these diagonal curves will be the families of equipotentials and stream lines. Analytically, this last result means that the determination of the stream lines depends upon two arbitrary functions of one real variable. It is shown that the angle between the characteristics and the angle formed by a tangent to a stream line and the $x$-axis can be determined in terms of these functions. Further, the magnitude of the velocity and the density depend upon only the former angle and the Mach number of the flow. In particular, if a known stream line coincides with the $x$-axis, it is shown that only one arbitrary function enters into the problem of determining the stream lines. Even in this last case where the data are of a simple Dirichlet type (symmetric flow about the $x$-axis and a known external boundary stream line-as in the jet problem), the direct problem cannot be solved easily. Hence, an analytical-geometrical method is outlined for solving the problem indirectly. A particular example is studied. Finally, in an appendix, we furnish another proof (analytical) of the fact that when the Kármán-Tsien relation is applicable, the characteristics form a Tschebyscheff net and conversely.

2. Extension of the Kármán-Tsien method to supersonic flow. In this section, we shall show that the Kármán-Tsien method may be extended to the supersonic flow of a perfect, irrotational, compressible fluid. If we denote the pressure by $p$, the density by $\rho$, the ratio of the specific heats by $\gamma$, the adiabatic relation is

$$
p \rho^{-\gamma}=\text { constant. }
$$

* Received Oct. 16, 1944.

1 T. von Kármán, Compressibility effects in aerodynamics, Journal of Aeron. Sciences 8, 337-356 (1941).

H. S. Tsien, Two-dimensional subsonic flows of compressible fluids, Journal of Aeron. Sciences 6, 399-407 (1939).

2 L. Bianchi, Lezioni di geometria differentiale, vol. 1, Enrico Spoerri, Pisa, 1922, pp. 153-162. 
Replacing the isentropic curve $(2.1)$ by the tangent line drawn at the point $\left(\rho_{1}^{-1}, p_{1}\right)$ in the pressure-volume diagram (or by a hyperbola drawn at the point $\left(\rho_{1}, p_{1}\right)$ in the pressure-density diagram), Tsien obtains the relation

$$
p_{1}-p=a_{1}^{2} \rho_{1}^{2}\left(\rho^{-1}-\rho_{1}^{-1}\right),
$$

where $a_{1}^{2}$ is the velocity of sound corresponding to $\left(\rho_{1}, p_{1}\right)$. By use of $(2.2)$, it is easily shown that the Bernoulli relation becomes (where $w$ is the velocity)

$$
w^{2}-w_{1}^{2}=a_{1}^{2} \rho_{1}^{2}\left(\rho^{-2}-\rho_{1}^{-2}\right) .
$$

Further, by use of (2.2) and the definition of $a^{2}$ (that is, $a^{2}=d p / d \rho$ ), it follows that

$$
a^{2} \rho^{2}=a_{1}^{2} \rho_{1}^{2}=k^{2}
$$

where $k$ is some constant. Hence, (2.3) can be transformed into the following forms:

$$
\left(\frac{w}{a_{1}}\right)^{2}-\left(\frac{w_{1}}{a_{1}}\right)^{2}=\left(\frac{\rho_{1}}{\rho}\right)^{2}-1
$$

$$
w^{2}-a^{2}=w_{1}^{2}-a_{1}^{2}=l^{2},
$$

where $l$ is some constant.

In the following, we shall assume that the point $\left(\rho_{1}, p_{1}\right)$ corresponds to a supersonic state of the fluid. From (2.5), we see that as $w$ increases, $\rho$ decreases. Further, from (2.4), we see that as $\rho$ decreases, $a$ increases. As noted by Tsien, the first result is in accord with the physical facts; the second result is in contradiction to known physical facts. However, (2.6) furnishes some useful information. Since the density $\rho_{1}$ corresponds to a supersonic state of the fluid, the equation (2.1) is valid for this $\rho_{1}$ and the corresponding $p_{1}$. Hence, by well known results, $w_{1}$ is larger than $a_{1}$. Thus from (2.6), we see that $w$ is always larger than $a$. That is, the fluid is always in a supersonic state in this sense of the term. However, by dividing (2.6) by $a^{2}$ and noting that as $w$ increases, $\rho$ decreases, and $a$ increases, we see that as $w$ increases, $w / a$ decreases. This ratio approaches the limiting value 1 as $w$ tends to infinity. Hence, the behavior of $w / a$ is contrary to that of a real fluid.

Perhaps the best indication of the permissible values of $w$ which can be used for a given $w_{1}$ is obtained by following the procedure of Tsien. If we consider the upper limit of the useful values of $w$ as occurring for $p=0$, we find that the corresponding $\rho$ is given by

$$
\frac{1}{\rho}=\frac{p_{1}+a_{1}^{2} \rho_{1}}{a_{1}^{2} \rho_{1}^{2}} .
$$

Substituting this value of $\rho$ into (2.3), we obtain the relation

$$
\left(\frac{w}{w_{1}}\right)^{2}=1+\frac{1}{\left(w_{1} / a_{1}^{2}\right)}\left[\left(\frac{p_{1}}{a_{1}^{2} \rho_{1}}+1\right)^{2}-1\right] .
$$

Since the values of $\rho_{1}, p_{1}$ satisfy (2.1), we find

$$
a_{1}^{2}=\left(\frac{d p}{d \rho}\right)_{1}=\frac{\gamma p_{1}}{\rho_{1}} .
$$


Thus (2.8) becomes

$$
\left(\frac{w}{w_{1}}\right)^{2}=1+\frac{1}{\left(w_{1} / a_{1}\right)^{2}}\left[\left(\frac{1}{\gamma}+1\right)^{2}-1\right] .
$$

If $\gamma$ is taken as 1.4 , a simple computation reveals that as $w_{1} / a_{1}$ goes from 1 to $\infty$, the ratio $w / w_{1}$ runs from 1.7 to 1 . That is, for large values of the ratio $w_{1} / a_{1}$, the range of applicability of formula (2.2) is severely restricted as regards the upper limit of $w$. Hence, the Kármán-Tsien relation should be useful in the supersonic range for a fairly uniform fluid flow. Further, as we shall show in the next section, the characteristics in this case form a Tschebyscheff (fish) net. We shall not be concerned with the onset of shocks.

3. The geometry of the characteristics for the relation (2.2). If $u(x, y)$ and $v(x, y)$ denote, respectively, the $x$ and $y$ components of the velocity for the steady flow of a fluid at any point $P$ of the plane region considered and $\rho(x, y)$ denotes the density of the fluid at $P$, then from the equation of continuity it follows that a stream function $\psi(x, y)$ exists such that

$$
\rho u=\frac{\partial \psi}{\partial y}, \quad \rho v=-\frac{\partial \psi}{\partial x} .
$$

Further, since the motion is irrotational, a velocity potential exists such that

$$
u=\frac{\partial \phi}{\partial x}, \quad v=\frac{\partial \phi}{\partial y} .
$$

For a given pressure-density relation, the Bernoulli relation determines $\rho$ as a function of $u^{2}+v^{2}$. Hence (3.1), (3.2) constitute a non-linear system. Eliminating the partial derivatives of $\rho$ from the continuity relation by use of the Euler equations, we find that $\phi(x, y)$ satisfies $^{3}$

$$
\left(a^{2}-u^{2}\right) \frac{\partial^{2} \phi}{\partial x^{2}}-2 u v \frac{\partial^{2} \phi}{\partial x \partial y}+\left(a^{2}-v^{2}\right) \frac{\partial^{2} \phi}{\partial y^{2}}=0 .
$$

In the supersonic range, the equation (3.3) is hyperbolic. Let us denote the equations of the two parameter family of characteristics of (3.3) by

$$
x=x(\alpha, \beta), \quad y=y(\alpha, \beta),
$$

where $\alpha=$ constant and $\beta=$ constant are the parametric equations of the characteristics. We denote the arc length element of the net formed by the characteristics by

$$
d s^{2}=A^{2}(d \alpha)^{2}+B^{2}(d \beta)^{2}+C d \alpha d \beta,
$$

where $A^{2}, B^{2}, C$ are the metric coefficients of the net. It follows from (3.3) that the projections of the velocity vector on the normals to the characteristics have the magnitude $a$. This means that the projections of a vector, normal to the velocity vector and of magnitude equal to that of the velocity vector, on the tangents to the characteristics have the magnitude $a$. Also, the projections of the velocity vector on the tangents to the characteristics have the magnitude $\sqrt{u^{2}+v^{2}-a^{2}}$. Further, from p. 230.

${ }^{3}$ R. von Mises and K. O. Friedrichs, Fluid dynamics, Brown University, Providence, R. I., 1941, 
(3.1), it follows that $\rho^{-1}$ times the gradient of $\psi$ is a vector, normal to the velocity vector and of magnitude equal to that of the velocity vector; and, from (3.2), it follows that the gradient of $\phi$ is the velocity vector. From the above properties of the characteristics and those of the gradient, it follows that

$$
\begin{array}{ll}
\frac{\partial \psi}{\partial \alpha}=\rho a A, & \frac{\partial \psi}{\partial \beta}=-\rho a B, \\
\frac{\partial \phi}{\partial \alpha}=\sqrt{u^{2}+v^{2}-a^{2}} A, & \frac{\partial \phi}{\partial \beta}=\sqrt{u^{2}+v^{2}-a^{2}} B .
\end{array}
$$

We shall prove that the net of characteristics forms a Tschebyscheff net, when the Karman-Tsien relation is applicable.

From (2.4), (2.6) and (3.6), (3.7), we find

$$
\frac{\partial \psi}{\partial \alpha}=k A, \frac{\partial \psi}{\partial \beta}=-k B,
$$

$$
\frac{\partial \phi}{\partial \alpha}=l A, \quad \frac{\partial \phi}{\partial \beta}=l B .
$$

The integrability conditions for (3.8), (3.9) furnish the result

$$
\frac{\partial A}{\partial \beta}=\frac{\partial B}{\partial \alpha}=0 .
$$

Hence $A$ and $B$ are functions of $\alpha$ and $\beta$, respectively. By proper choice of scale factors, $A$ and $B$ may be assigned the value unity. The new parameters $\alpha$ and $\beta$ are then arc length parameters and the net is a Tschebyscheff net.

Next, we shall derive a result similar to that obtained by von $\mathrm{Mises}^{4}$ in plane plasticity: when the Karman-Tsien relation is applicable and the diagonal curves of the characteristics are drawn so as to correspond to equi-distant values of the arc length parameter along the characteristics, then these diagonal curres will be the families of equipotentials and stream lines.

Since (2.2) is valid, we see from our previous result that $\alpha$ and $\beta$ may be chosen as arc length parameters. Hence, the nets

$$
\alpha+\beta=2 \xi=\text { constant }, \quad \alpha-\beta=2 \eta=\text { constant },
$$

represent, respectively, the diagonal curves of the net of characteristics, corresponding to equi-intervaled values of the arc length parameters $\alpha$ and $\beta$. Further, from (3.8), (3.9), we obtain

$$
\alpha+\beta=\frac{\phi}{l}, \quad \alpha-\beta=\frac{\psi}{k} .
$$

Hence, the diagonal curves $\xi=$ constant and $\eta=$ constant represent, respectively, the equipotentials and stream lines.

With the aid of our previous results and known properties of Tschebyscheff nets, we obtain some additional results. The general representation of the stream lines in the supersonic range for the Karman-Tsien relation depends upon two real arbitrary functions. If one stream line coincides with the $x$-axis, these two functions are equal except for

${ }^{4}$ R. von Mises, Bemerkung zur Formulierung des mathematischen Problems der Plastizitätstheorie, Zeitschr. für angew. Math. u. Mech., 5, 147-149 (1925). 
a constant. Further, the velocity and density depend only upon the angle between the characteristics and the Mach number of the flow.

For a Tschebyscheff net, it is well known ${ }^{2}$ that (3.5) may be written in the form

$$
d s^{2}=(d \alpha)^{2}+2 \cos \omega d \alpha d \beta+(d \beta)^{2},
$$

where $\omega$ is the angle between the two families of characteristics of the net at any point. Further, it is known that $\omega$ may be expressed in terms of two arbitrary functions $F(\alpha)$ and $G(\beta)$ by the relation

$$
\omega=F(\alpha)+G(\beta) \text {. }
$$

Finally, the general representation of the net is given by

$$
\begin{aligned}
& x=\int^{\alpha} \cos F(t) d t+\int^{\beta} \cos G(t) d t, \\
& y=\int^{\alpha} \sin F(t) d t-\int^{\beta} \sin G(t) d t .
\end{aligned}
$$

Introducing the parameters along the equipotentials and stream lines from (3.11), we find that the above equations become

$$
\begin{aligned}
d s^{2} & =4 \cos ^{2} \frac{\omega}{2}(d \xi)^{2}+4 \sin ^{2} \frac{\omega}{2}(d \eta)^{2}, \\
\omega & =F(\xi+\eta)+G(\xi-\eta), \\
x & =\int^{\xi+\eta} \cos F(t) d t+\int^{\xi-\eta} \cos G(t) d t, \\
y & =\int^{\xi+\eta} \sin F(t) d t-\int^{\xi-\eta} \sin G(t) d t .
\end{aligned}
$$

Another relation of the form (3.18) can be obtained by introducing the angle $\theta(\xi, \eta)$ which the tangents to the stream lines $(\eta=$ constant) form with the $x$-axis. Let the equations of a stream line be

$$
x=x(s), \quad y=y(s), \quad \eta=\text { constant },
$$

where $s$ is the arc length parameter along the stream line. From (3.19), we find by differentiation

$$
\frac{d x}{d s}=[\cos F(\xi+\eta)+\cos G(\xi-\eta)] \frac{d \xi}{d s} .
$$

By use of the well known addition formulas of trigonometry, (3.22) becomes

$$
\frac{d x}{d s}=2 \cos \left[\frac{F(\xi+\eta)+G(\xi-\eta)}{2}\right] \cos \left[\frac{F(\xi, \eta)-G(\xi-\eta)}{2}\right] \frac{d \xi}{d s} .
$$

From (3.17), we find that along $\eta=$ constant

$$
\frac{d \xi}{d s}=\frac{1}{2} \sec \left[\frac{F(\xi+\eta)+G(\xi-\eta)}{2}\right] \text {. }
$$


Substituting (3.24) into the right-hand side of (3.23), we obtain

$$
\frac{d x}{d s}=\cos \left[\frac{F(\xi+\eta)-G(\xi-\eta)}{2}\right] \text {. }
$$

From (3.25), we find that except for a constant

$$
2 \theta=F(\xi+\eta)-G(\xi-\eta) .
$$

By use of (3.14), (3.15), (3.16), and (3.26), the magnitude of the velocity $w$ and the density $\rho$ may be shown to be expressible solely in terms of $\omega$ and the Mach number of the flow. Thus, from (3.2), (3.12)

$$
u=l\left(\frac{\partial \alpha}{\partial x}+\frac{\partial \beta}{\partial x}\right), \quad v=l\left(\frac{\partial \alpha}{\partial y}+\frac{\partial \beta}{\partial y}\right) .
$$

Hence, by interchanging the independent and dependent variables, it follows that

$$
u=\frac{l}{D}\left(\frac{\partial y}{\partial \beta}-\frac{\partial y}{\partial \alpha}\right), \quad v=\frac{l}{D}\left(\frac{\partial x}{\partial \alpha}-\frac{\partial x}{\partial \beta}\right),
$$

where

$$
D=\frac{\partial x}{\partial \alpha} \frac{\partial y}{\partial \beta}-\frac{\partial x}{\partial \beta} \frac{\partial y}{\partial \alpha} .
$$

Computing the partial derivatives by use of (3.15), (3.16) and simplifying by use of (3.14), (3.26), we obtain

$$
u=2 l \sin \frac{1}{2} \omega \cos \theta / \sin \omega, \quad v=2 l \sin \frac{1}{2} \omega \sin \theta / \sin \omega .
$$

Hence, for the magnitude $w$ of the velocity, we find

$$
w=\frac{2 l \sin \frac{1}{2} \omega}{\sin \omega} \text {. }
$$

From (2.6), we see that $l^{2}$ is equal to $w_{1}^{2}-a_{1}^{2}$. Making this substitution in (3.31) and dividing the resulting equation by $w_{1}$, we obtain

$$
\frac{w}{w_{1}}=\frac{2 \sqrt{1-\left(a_{1} / w_{1}\right)^{2}} \sin \frac{1}{2} \omega}{\sin \omega} .
$$

In Fig. 1, these curves are plotted for the following values of the Mach number, $w_{1} / a_{1}=1.5,2.0,2.5,3.0,4.0$. Note, by the discussion following equation (2.10), as $w_{1} / a_{1}$ varies from 1 to $\infty$, the permissible values of the upper bound of $w / w_{1}$ varies from 1.7 to 1 . In each case, the upper bounds are to be determined by use of $(2.10)$. The dotted lines in Fig. 1 denote these upper bounds. Other useful results may be obtained by combining (3.31) with (2.6) and (2.3). Thus, dividing the equation $w^{2}-a^{2}=l^{2}$ by $w^{2}$ and inserting the value of $w$ as determined from (3.31) into the right-hand member of the resulting equation, we find after a few trigonometric substitutions

$$
\frac{w}{a}=\frac{1}{\sin \frac{1}{2} \omega} .
$$


This equation is of value in determining the lower limit of the ratio $w / a$, namely, $\sqrt{2}$, for $\omega=\pi / 2$. Again, inserting the value of $w$ as determined from (3.31) into the left-hand side of (2.3), dividing the resulting equation by $a_{1}^{2}$, and replacing the term $l^{2} / a_{1}^{2}$ by $w_{1}^{2} / a_{1}^{2}-1$ (see 2.6 ), we obtain

$$
\frac{\rho}{\rho_{1}}=\frac{\sin \omega}{2 \sin ^{2} \frac{1}{2} \omega \sqrt{\left(w_{1} / a_{1}\right)^{2}-1}} .
$$

These curves are plotted in Fig. 2 for the values of the Mach number as indicated above.

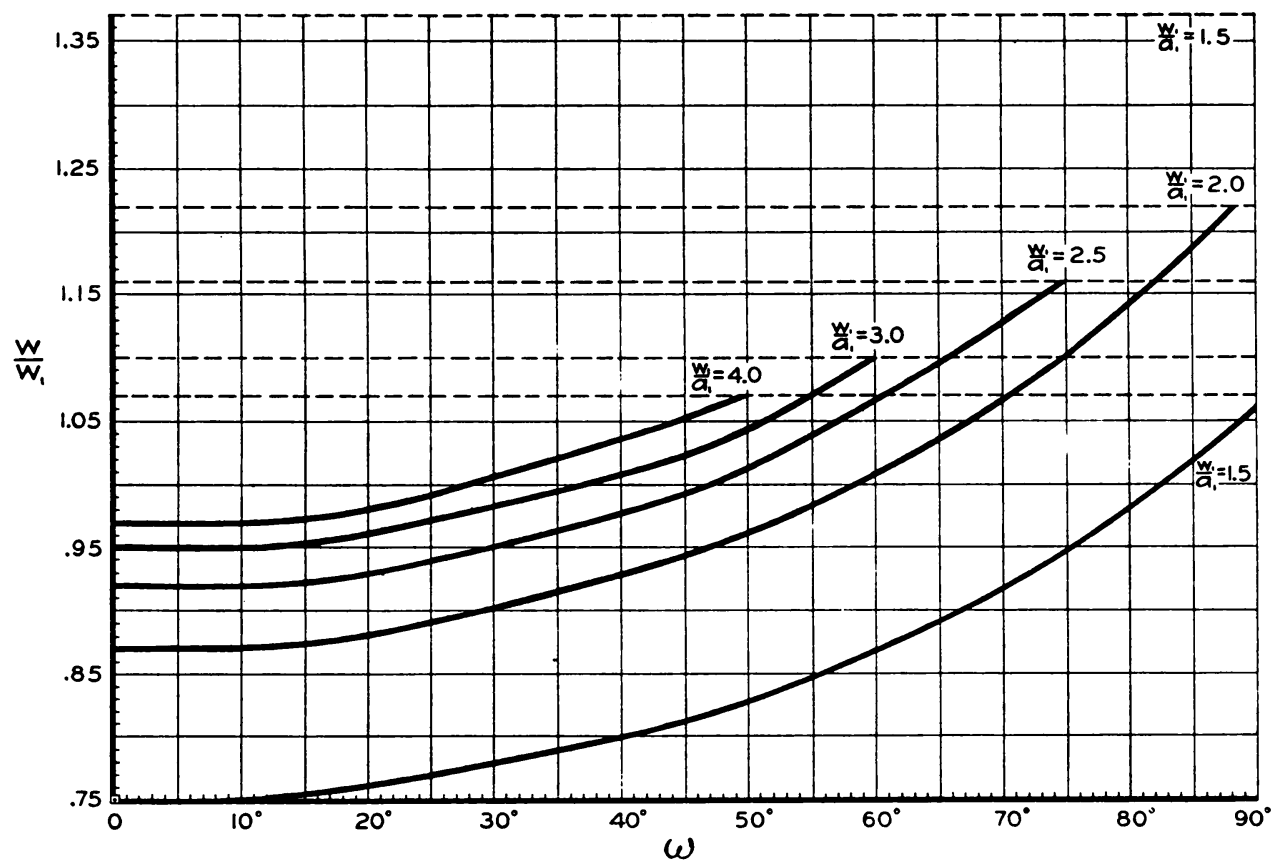

FIG. 1 .

An important case in practice ${ }^{5}$ (the jet problem) is that for which one stream line is a straight line. In this case, if we assume that the stream line coincides with the $x$-axis and is $\eta=0,(3.26)$ furnishes the result

$$
F(\xi)=G(\xi) .
$$

Under properly given Dirichlet data, the function $F(\xi)$ can be determined and the representation of all stream lines can be obtained from (3.19), (3.20). Thus, if $\theta(\xi, c)$ is known along some known stream line $\eta=c$, then the equations (3.26) and (3.35) furnish the result

$$
2 \theta(\xi, c)=F(\xi+c)-F(\xi-c) .
$$

The equation (3.36) can be solved for $F(\xi)$ by use of the theory of difference equations.

'J. Ackeret, Gasdynamik, Handbuch der Physik, vol. 7. pp. 318-322. 
Unfortunately, $\theta(\xi, c)$ is unknown; $\theta(s)$, where $s$ is the arc length parameter, is known. Hence, one must solve problems by an indirect method. That is, one must introduce a function $F(\xi)$ and then determine the corresponding stream lines.

In calculating the stream lines for particular functions $F(\xi)$, the following analytical-geometrical scheme appears to be the most satisfactory. First, obtain two curves

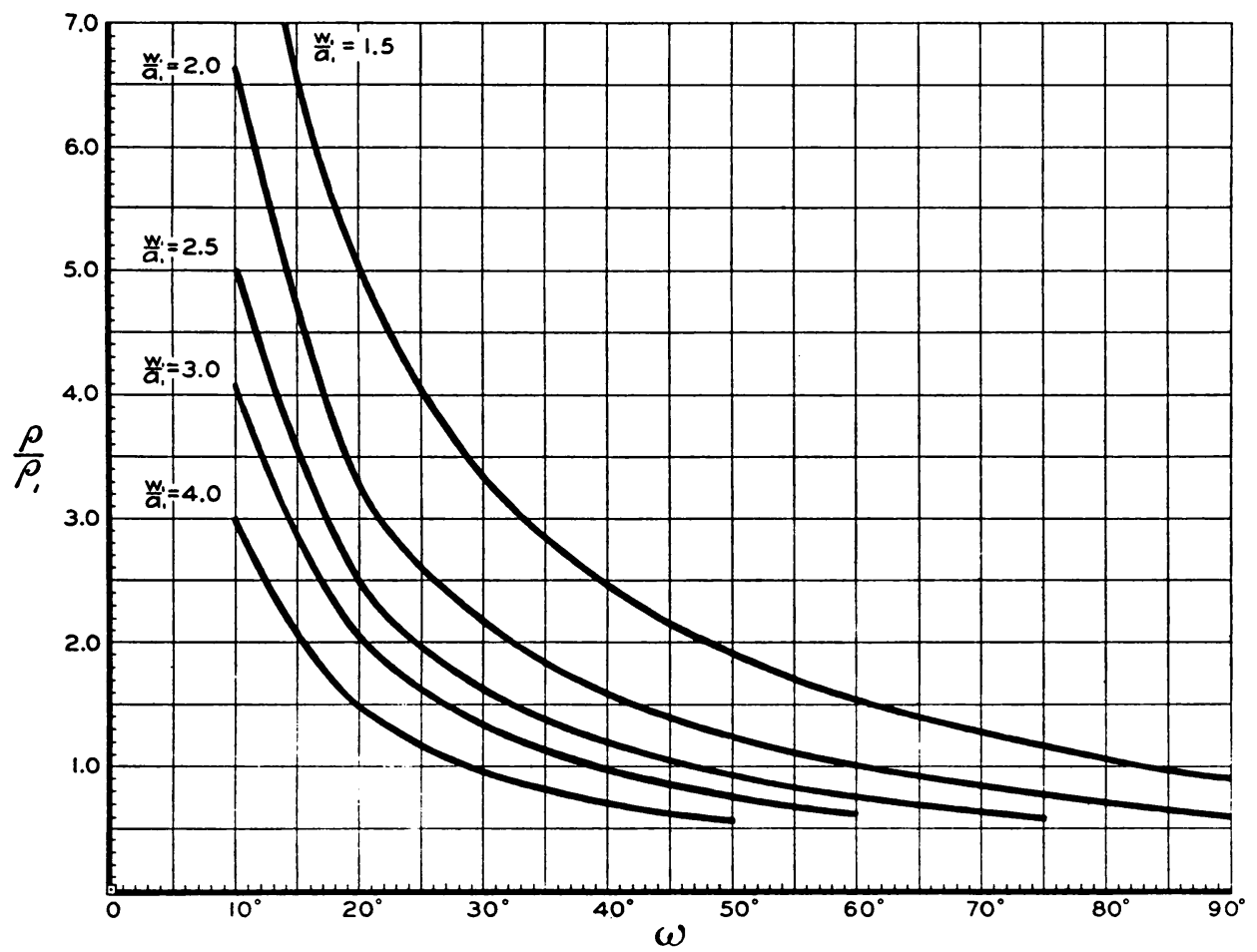

FIG. 2.

of the generating Tschebyscheff net (one of each family) by use of equations (3.15), (3.16). That is, determine the curves

$$
\begin{array}{ll}
x_{1}=\int^{\alpha} \cos F(t) d t, & y_{1}=\int^{\alpha} \sin F(t) d t, \\
x_{2}=\int^{\beta} \cos F(t) d t, & y_{2}=-\int^{\beta} \sin F(t) d t .
\end{array}
$$

To obtain the initial point of each curve in the $x, y$ plane, we compute one set of values of $(x, y)$ by use of (3.15), (3.16), for some set of values of $(\alpha, \beta)$ such as $\alpha=0$, $\beta=0$. By translating the curves along each other, the complete Tschebyscheff net may be obtained. However, the translation must furnish curves of the families which correspond to equi-distant values of $\alpha$ and $\beta$ in order that the diagonal curves be stream lines. In view of (3.15), (3.16), this means that the abscissas of the initial points of two corresponding curves must be equal. 
Finally, as an example of this method, let us consider the case $F(t)=\arccos t$. From (3.37), (3.38), it follows that

$$
\begin{aligned}
& x_{1}=\frac{\alpha^{2}}{2}, \quad y_{1}=\frac{\alpha}{2} \sqrt{1-\alpha^{2}}+\frac{1}{2} \arcsin \alpha, \\
& x_{2}=\frac{\beta^{2}}{2}, \quad y_{2}=-\frac{\beta}{2} \sqrt{1-\beta^{2}}-\frac{1}{2} \arcsin \beta .
\end{aligned}
$$

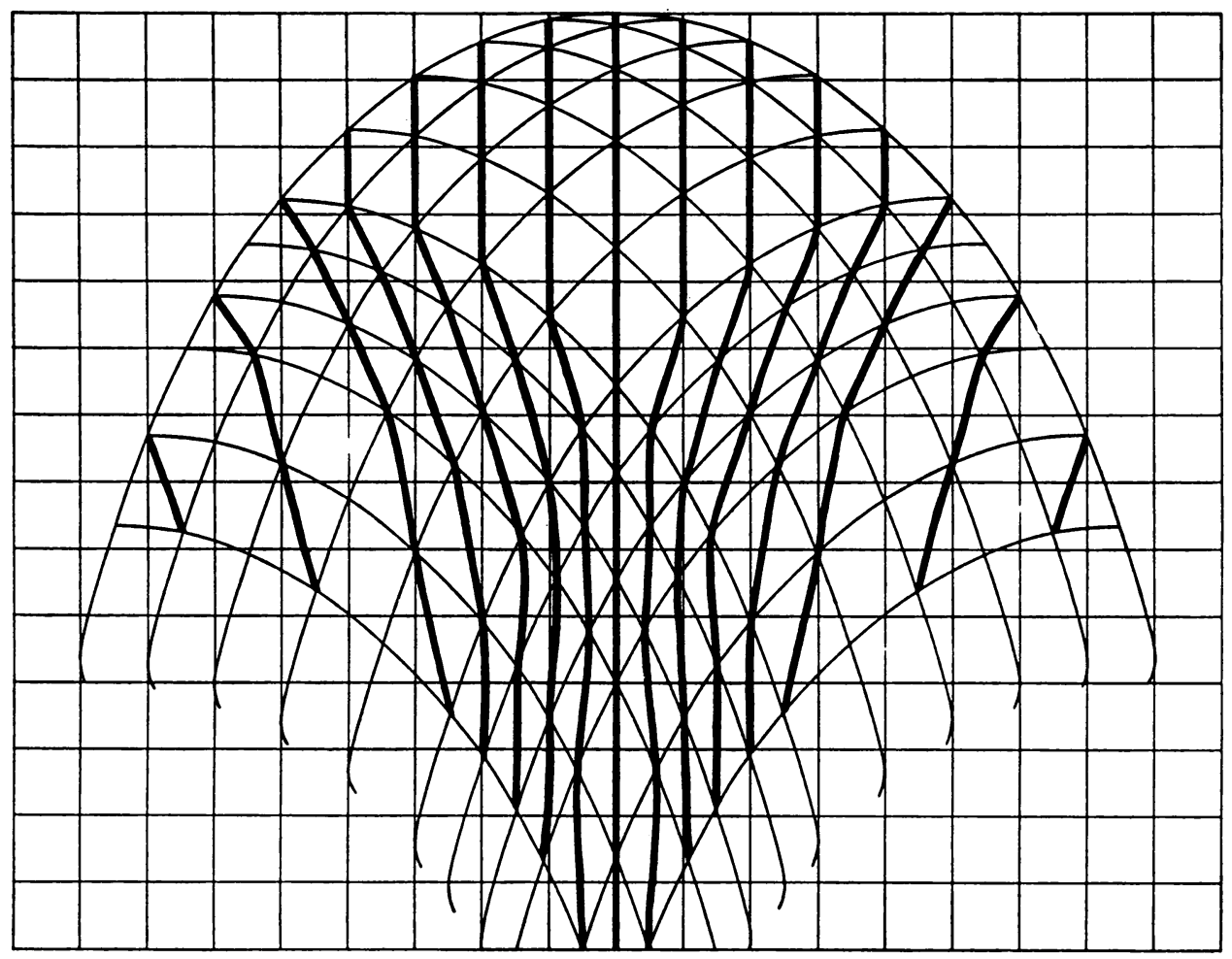

FIG. 3.

The Tschebyscheff net and the resulting stream lines, obtained by the procedure outlined in the preceding paragraph, are illustrated in Fig. 3. By use of a protractor and the graphs of Figs. 1 and 2, the values of $w / w_{1}$ and $\rho / \rho_{1}$ can be immediately determined at each point of the plane.

In concluding, it should be pointed out that it would be highly desirable to obtain a mechanical method for constructing the Tschebyscheff net when two stream lines are known. This would furnish a direct solution to the problem of the uniform flow of a supersonic jet. It appears that a more thorough understanding of the relation between Tschebyscheff nets and their diagonal curves is needed. 


\section{APPENDIX}

First, we shall give an analytic derivation of relations (3.6), (3.7). Since the projections of the velocity vector on the normal to the characteristics have the magnitude $a$, it follows that

$$
\begin{aligned}
a A & =u \frac{\partial y}{\partial \alpha}-v \frac{\partial x}{\partial \alpha}, \\
-a B & =u \frac{\partial y}{\partial \beta}-v \frac{\partial x}{\partial \beta} .
\end{aligned}
$$

Further, by projecting the velocity vector on the tangents to the characteristics, we obtain

$$
\begin{aligned}
& \sqrt{u^{2}-v^{2}-a^{2}} A=u \frac{\partial x}{\partial \alpha}+v \frac{\partial y}{\partial \alpha}, \\
& \sqrt{u^{2}+v^{2}-a^{2}} B=u \frac{\partial x}{\partial \beta}+v \frac{\partial y}{\partial \beta} .
\end{aligned}
$$

Solving relations (A.1), (A.2) and (A.3), (A.4) for $u, v$, we find

$$
\begin{aligned}
& u=-\frac{a}{D}\left(B \frac{\partial x}{\partial \alpha}+A \frac{\partial x}{\partial \beta}\right)=\frac{\sqrt{u^{2}+v^{2}-a^{2}}}{D}\left(A \frac{\partial y}{\partial \beta}-B \frac{\partial y}{\partial \alpha}\right), \\
& v=-\frac{a}{D}\left(B \frac{\partial y}{\partial \alpha}+A \frac{\partial y}{\partial \beta}\right)=\frac{\sqrt{u^{2}+v^{2}-a^{2}}}{D}\left(B \frac{\partial x}{\partial \alpha}-A \frac{\partial x}{\partial \beta}\right),
\end{aligned}
$$

where $D$ is the Jacobian of the transformation (3.4). By interchanging dependent and independent variables, (A.5), (A.6) become

$$
\begin{aligned}
& u=a\left(A \frac{\partial \alpha}{\partial y}-B \frac{\partial \beta}{\partial y}\right)=\sqrt{u^{2}+v^{2}-a^{2}}\left(A \frac{\partial \alpha}{\partial x}+B \frac{\partial \beta}{\partial x}\right), \\
& v=-a\left(A \frac{\partial \alpha}{\partial x}-B \frac{\partial \beta}{\partial x}\right)=\sqrt{u^{2}+v^{2}-a^{2}}\left(A \frac{\partial \alpha}{\partial y}+B \frac{\partial \beta}{\partial y}\right) .
\end{aligned}
$$

From (A.7), (A.8) and (3.2), we find (3.6), (3.7).

Next, we shall show that the Karman-Tsien relation (2.2) is valid, if the net of characteristics form a Tschebyscheff net.

Since the net of characteristics is a Tschebyscheff net, we may consider the metric coefficients $A$ and $B$ as having the value unity. Hence, from (3.6), (3.7) and the chain rule for differentiation, we obtain

$$
\begin{array}{ll}
\frac{\partial \psi}{\partial x}=\rho a \frac{\partial(\alpha-\beta)}{\partial x}, & \frac{\partial \psi}{\partial y}=\rho a \frac{\partial(\alpha-\beta)}{\partial y}, \\
\frac{\partial \phi}{\partial x}=\sqrt{u^{2}+v^{2}-a^{2}} \frac{\partial(\alpha+\beta)}{\partial x}, & \frac{\partial \phi}{\partial y}=\sqrt{u^{2}+v^{2}-a^{2}} \frac{\partial(\alpha+\beta)}{\partial y} .
\end{array}
$$


With the aid of (3.1), (3.2), we may write the integrability conditions of (A.9), (A.10) in the form

$$
\frac{\partial}{\partial s} \rho a=0
$$

$$
\frac{\partial}{\partial n} \sqrt{u^{2}+v^{2}-a^{2}}=0,
$$

where $\partial / \partial s$ represents differentiation along a stream line and $\partial / \partial n$ represents differential along an equipotential. If we assume that a relation exists between $p$ and $\rho$, then by use of the definition of $a^{2}$ (defined as $d p / d \rho$ ), we find from (11)

$$
\rho \frac{d^{2} p}{d \rho^{2}}+2 \frac{d p}{d \rho}=0 .
$$

Further, from the generalized Bernoulli relation

$$
u^{2}+v^{2}+2 P(\rho)=\text { constant }, \quad \frac{d P}{d \rho}=\frac{1}{\rho} \frac{d p}{d \rho},
$$

we find that (A.12) reduces to (A.13). Integrating (A.13), we obtain (2.2).

The author wishes to thank Professor W. Prager and Dr. L. Bers of Brown University for valuable criticisms and suggestions. Further, he is indebted to the Research Institute of the University of Texas for a grant which permitted the construction of the diagrams. 